\title{
QUALIDADE MICROBIOLÓGICA E FÍSICO-QUÍMICA DE ALMÔNDEGAS ELABORADAS COM CARNE DE CAPIVARA E DIFERENTES CONCENTRAÇÕES DE BACON
}

\author{
Gabriella Castro Cunha ${ }^{1}$, Vilson Matias Pinto ${ }^{1}$, Brena Cristine Rosário Silva ${ }^{1}$, Diego da Silva Pedro ${ }^{1}$, \\ Márcio Ramatiz Lima dos Santos ${ }^{2}$, Waldeliza Fernandes da Cunha ${ }^{3}$. \\ ${ }^{1}$ Estudantes do curso de Zootecnia, Instituto Federal Goiano - Campus Ceres, Bacharelado em Zootecnia. ${ }^{2}$ Professor \\ do Curso de Zootecnia, Instituto Federal Goiano - Campus Ceres. ${ }^{3}$ Professora do Curso de Zootecnia, Instituto Federal \\ Goiano - Campus Ceres. E-mail: gabriellah castro@hotmail.com
}

\section{RESUMO}

Objetivou-se avaliar a qualidade das almôndegas elaboradas com carne de capivara e diferentes concentrações de bacon suíno. Foram elaboradas três formulações de almôndegas da capivara sendo uma com $100 \%$ de carne de capivara e as demais com adição de 10 e $20 \%$ de bacon suíno. Foram realizadas Coliformes a 35 e a 45 으 C das amostras de almôndegas, além das determinações de $\mathrm{pH}$, acidez titulável, umidade e cinzas. Em relação às determinações físico-químicas, os resultados foram submetidos à análise de variância e as diferenças das médias comparadas por teste de Tukey ao nível de $5 \%$ de significância. Os resultados das análises microbiológicas foram avaliados de forma descritiva. Foi utilizado o delineamento experimental inteiramente casualizado. Todas as determinações físico-químicas e microbiológicas, nas amostras foram realizadas em triplicata. As análises de coliformes mostraram que as almôndegas estavam aptas ao consumo e os resultados físico-químicos estão em níveis aceitáveis.

Palavras-chave: animais silvestres; mercado; qualidade.

\section{MICROBIOLOGICAL AND PHYSICAL-CHEMICAL QUALITY OF ALMÔNDEGAS ELABORATED WITH CAPITAL MEAT AND DIFFERENT BACON CONCENTRATIONS}

\begin{abstract}
The objective of this study was to evaluate the quality of meatballs prepared with capybara meat and different concentrations of pork bacon. Three formulations of capybara meatballs were prepared: one with $100 \%$ of capybara meat and the other with 10 and $20 \%$ of pork bacon added. Coliforms were carried out at 35 and $45^{\circ} \mathrm{C}$ of the meatball samples, in addition to $\mathrm{pH}$, titratable acidity, moisture and ash determinations. In relation to the physical-chemical determinations, the results were submitted to analysis of variance and the differences of the means compared by Tukey test at the $5 \%$ level of significance. The results of the microbiological analyzes were evaluated in a descriptive way. The experimental design was completely randomized. All physicalchemical and microbiological determinations were performed in triplicate. Coliform analyzes showed that the meatballs were fit for consumption and the physicochemical results are in acceptable levels.
\end{abstract}

Keywords: wild animals; marketplace; quality.

\section{INTRODUÇÃO}

A capivara (Hydrochoerus hydrochaeris) é um dos principais representantes da ordem Rodentia. Pertencente à família Hydrochaeridae, a espécie é considerada o maior roedor vivente. 
É um herbívoro generalista de hábito semiaquático, cuja utilização do espaço no ambiente natural está diretamente relacionada à disponibilidade alimentar (FELIX, 2012).

Segundo Rebello et al. (2002), a exploração de animais silvestres em criatórios autorizados, associada a exploração pecuária e agrícola convencional resulta em benefícios ambientais, sociais e econômicos. Essa atividade tem despertado atenção dos proprietários rurais devido a seu baixo custo de produção e a existência de um amplo mercado para seus produtos.

A carne da capivara tem boa aceitação comercial principalmente por ser muito saborosa, apresentando ainda qualidades bastante procuradas por certas camadas da população brasileira, como alto teor proteico, baixa porcentagem de gordura e colesterol, se comparado as carnes de bovino, suíno e frango (SILVA, 2013).

Uma pesquisa realizada por Frasson e Salgado (1990) mostrou que a carne de capivara apresenta seu teor de gordura mais baixo do que a carne bovina. A pesquisa ainda mostra que os teores de proteínas na carne de capivara (lombo, traseiro e dianteiro) são 12,63\% superiores aos da carne bovina (alcatra) e 11,67\% superiores aos de carne suína (lombo, traseiro e dianteiro).

No Brasil, o mercado apresenta-se promissor, pois a procura pela carne e pele é incomparavelmente maior que a oferta (ROCHA, 2003). A carne de capivara é consumida em todo o país, especialmente na Amazônia, onde é uma das mais apreciadas pelos povos indígenas (JARDIM, 2001).

Objetivou-se com esse trabalho avaliar qualidade microbiológica e físico-química de almôndegas elaboradas a partir da carne de capivara com diferentes concentrações de bacon suíno.

\section{MATERIAL E MÉTODOS}

A carne de capivara foi doada pela Prof. Dra. Waldeliza Fernandes de Cunha, proveniente de um criatório comercial, em Jataí, GO, número do registro no IBAMA 38/2013, inscrição estadual 11085246-0.

Foram utilizadas três formulações diferentes para o preparo das almôndegas (Tabela 1), sendo: uma das formulações com apenas carne de capivara ( $0 \%$ de bacon), e as outras duas formulações foram adicionadas $10 \%$ e $20 \%$ de bacon. Outros ingredientes, como sal, cebola, pimenta de cheiro e alho, também foram utilizados na mesma proporção em todas as receitas.

Tabela 1. Proporções dos ingredientes utilizados na fabricação das almôndegas de carne de capivara

\begin{tabular}{lccc}
\hline \multicolumn{1}{c}{ Ingredientes } & A1 & A2 & A3 \\
\hline Carne de capivara $(\mathrm{g})$ & 3.000 & 2.700 & 2.400 \\
Bacon $(\mathrm{g})$ & 0 & 300 & 600 \\
Cebola $(\mathrm{g})$ & 100 & 100 & 100 \\
Alho $(\mathrm{g})$ & 10 & 10 & 10 \\
Pimenta de Cheiro $(\mathrm{g})$ & 10 & 10 & 10 \\
Sal $(\mathrm{g})$ & 10 & 10 & 10 \\
\hline
\end{tabular}

Legenda: A1 - almôndegas de carne de capivara com 0\% de bacon; A2 - almôndegas de carne de capivara com 10\% de bacon; A3 - almôndegas de carne de capivara com $20 \%$ de bacon.

As análises microbiológicas foram realizadas no laboratório de Microbiologia do Instituto Federal Goiano - Campus Ceres. Realizaram-se contagens de coliformes a 35으 e a 45으 das almôndegas de carne de capivara na forma crua e cozida, segundo BRASIL (2001).

As determinações microbiológicas, nas amostras foram realizadas em triplicata e os resultados avaliados de forma descritiva. As análises físico-químicas foram realizadas no laboratório de Química e no laboratório Instrumental do Instituto Federal Goiano - Campus Ceres. 
Foram realizadas as determinações de $\mathrm{pH}$, umidade, cinzas e acidez titulável das amostras de almôndegas da carne de capivara, segundo BRASIL (2008). Os valores de pH foram obtidos utilizando-se um potenciômetro digital previamente calibrado. As análises de umidade foram feita por secagem em estufa, a $100^{\circ} \mathrm{C}$ e os teores de cinzas por incineração em mufla, a $650^{\circ} \mathrm{C}$, até obtenção de massa constante.

Em relação às determinações físico-químicas, os resultados serão submetidos à análise de variância e as diferenças das médias comparadas por teste de Tukey ao nível de $5 \%$ de significância, utilizando-se o programa ASSISTAT (2000).

\section{RESULTADOS E DISCUSSÃO}

Nas análises de qualidade microbiológica das almôndegas, não foram encontradas presença de coliformes nas almôndegas de carne de capivara, cozidas e cruas, respectivamente (Tabelas 2 e 3).

Tabela 2. Resultados das análises microbiológicas das almôndegas de carne de capivara cozida

\begin{tabular}{ccc}
\hline Tratamentos & Coliformes a 35C (NMP/g) & Coliformes a 45C 35C (NMP/g) \\
\hline A1 & $<3$ & $<3$ \\
A2 & $<3$ & $<3$ \\
A3 & $<3$ & $<3$ \\
\hline *Valor de referência & ---- & $5 \times 10^{2}$ \\
\hline
\end{tabular}

Legenda: A1 - almôndegas de carne de capivara com 0\% de bacon; A2 - almôndegas de carne de capivara com 10\% de bacon; A3 - almôndegas de carne de capivara com $20 \%$ de bacon.

Tabela 3. Resultados das análises microbiológicas das almôndegas de carne de capivara crua

\begin{tabular}{ccc}
\hline Tratamentos & Coliformes a 35C (NMP/g) & Coliformes a 45C (NMP/g) \\
\hline B1 & $<3$ & $<3$ \\
B2 & $<3$ & $<3$ \\
B3 & $<3$ & $<3$ \\
\hline *Valor de referência & ---- & $5 \times 10^{2}$
\end{tabular}

Legenda: B1 - almôndegas de carne de capivara com 0\% de bacon; B2 - almôndegas de carne de capivara com 10\% de bacon; B3 - almôndegas de carne de capivara com $20 \%$ de bacon.

Segundo Pardi et al. (1993), é grande a gama de microrganismos ocorrentes nas carnes por causa de sua complexa composição (proteínas, glicídios, lipídeos, vitaminas e sais minerais) e de seu elevado teor de umidade, sendo propício ao desenvolvimento microbiano. Podendo prejudicar a qualidade do produto e reduzir sua validade no mercado, a contaminação microbiana não controlada da carcaça pode trazer perigos à saúde humana.

As almôndegas de carne de capivara tanto crua quanto cozida apresentaram-se dentro dos padrões exigidos pela legislação, onde não foi encontrada a presença significativa de coliformes a 35 ㅇ $\mathrm{C}$ e a 45 으 em nenhuma das amostras e suas respectivas repetições, estando dentro do valor mínimo aceito, sendo consideradas aptas à comercialização e consumo pela população. Segundo Brasil (2001), os limites estabelecidos para produtos cárneos crus resfriados como as almôndegas, são de número mais provável (NMP) máximo de 5,0 × 10²/g de produto.

Para as almôndegas cozidas já se esperava a ausência de coliformes devido ao processo de cozimento da carne que ajuda a prevenir e diminuir a proliferação de microrganismos deteriorantes e também eliminar possíveis agentes causadores de doenças e intoxicações alimentares. No trabalho de Sarkis (2002), avaliando as condições microbiológicas de carnes de animais silvestres no município de São Paulo, realizou contagens de coliformes a 35ㄷ e a 45ㄷ $\mathrm{C}$ das carnes de capivara, javali e cateto, seus resultados mostraram que as amostras de carne dos três 
animais estavam dentro dos limites máximos permitidos de acordo com Brasil (2001), sendo todas elas próprias para consumo humano.

Nos tratamentos analisando, aspectos físico-químicos (Tabela 4 e 5) obteve-se um pH variando entre 7,21 e 6,85 para almôndegas cozida, e para cruas ficaram entre 7,08 e 6,83. Nas almôndegas que foram cozidas o pH das amostras A1, A2 e A3 apresentaram-se neutros. Para as almôndegas cruas (B1, B2 e B3), observa-se resultados parecidos com as amostras cozidas, onde o $\mathrm{pH}$ diminui conforme aumentou a quantidade de bacon (Tabela 5). Segundo Foletto (2007), o pH é uma determinação importante para caracterizar a deterioração do alimento e para determinar a escolha da embalagem adequada para o produto.

Nas amostras de almôndegas o maior valor de umidade e cinzas encontrado foi de $51,35 \%$ e $0,97 \%$, sendo que os tratamentos $A 1, A 2$ e A3 foram iguais estatisticamente, tanto para umidade quanto para cinzas (Tabela 4). Para as almôndegas cruas (Tabela 5), o tratamento B1 foi mais significante para umidade $(84,91 \%)$ e cinzas $(1,28 \%)$, porém, para acidez não houve diferença significativa entre os tratamentos B1, B2 e B3.

O teor de umidade do tratamento B1 (Tabela 5) foi cerca de $20-30 \%$ superior aos demais tratamentos devido a almôndega ser apenas de carne de capivara, já as almôndegas com alguma adição de bacon variaram em 5 a 10\% em seu teor de umidade de crua para cozida, mostrando que o bacon ajudou a diminuir o teor de umidade das almôndegas quando ainda estavam cruas.

Segundo Miguel (2002), que avaliando o teor de umidade do lombo, pernil e patela em cortes de capivara, encontrou valores de $75 \%, 76 \%$ e $77 \%$, respectivamente.

Tabela 4. Valores das análises físico-químicas das almôndegas de carne de capivara cozida

\begin{tabular}{ccccc}
\hline Tratamentos & $\mathbf{p H}$ & Umidade (\%) & Cinzas (\%) & Acidez (g/100g) \\
\hline A1 & $7,21 \mathrm{a}$ & $51,35 \mathrm{a}$ & $0,97 \mathrm{a}$ & $0,04 \mathrm{a}$ \\
A2 & $6,92 \mathrm{~b}$ & $50,61 \mathrm{a}$ & $0,91 \mathrm{a}$ & $0,03 \mathrm{a}$ \\
A3 & $6,85 \mathrm{~b}$ & $50,22 \mathrm{a}$ & $0,97 \mathrm{a}$ & $0,04 \mathrm{a}$ \\
\hline
\end{tabular}

Legenda: A1 - almôndegas de carne de capivara com 0\% de bacon; A2 - almôndegas de carne de capivara com 10\% de bacon; A3 - almôndegas de carne de capivara com 20\% de bacon. Médias seguidas de letras diferentes, na mesma coluna, diferem estatisticamente entre si $(p<0,05)$ pelo teste de Tukey.

Tabela 5. Valores das análises físico-químicas das almôndegas de carne de capivara crua

\begin{tabular}{ccccc}
\hline Tratamentos & pH & Umidade (\%) & Cinzas (\%) & Acidez (g/100g) \\
\hline B1 & $7,08 \mathrm{a}$ & $84,91 \mathrm{a}$ & $1,28 \mathrm{a}$ & $0,03 \mathrm{a}$ \\
B2 & $6,88 \mathrm{~b}$ & $61,35 \mathrm{~b}$ & $0,97 \mathrm{ab}$ & $0,02 \mathrm{a}$ \\
B3 & $6,83 \mathrm{c}$ & $45,69 \mathrm{c}$ & $0,70 \mathrm{~b}$ & $0,03 \mathrm{a}$ \\
\hline
\end{tabular}

Legenda: B1 - almôndegas de carne de capivara com 0\% de bacon; B2 - almôndegas de carne de capivara com 10\% de bacon; B3 - almôndegas de carne de capivara com 20\% de bacon. Médias seguidas de letras diferentes, na mesma coluna, diferem estatisticamente entre si $(p<0,05)$ pelo teste de Tukey.

\section{CONCLUSÃO}

As almôndegas elaboradas com carne de capivara e diferentes concentrações de bacon mostraram uma boa condição higiênico-sanitária e estavam dentro dos padrões da legislação devido à ausência de coliformes a $35 \stackrel{\circ}{\circ}$ e a $45 \stackrel{\circ}{\circ}$. Os resultados físico-químicos estão em níveis aceitáveis para este tipo de alimento, apenas os teores de umidade variaram nas almôndegas cruas devido a relação dos teores de bacon, quanto maior o teor de bacon nas amostras de almôndega menor foi a umidade observada. Diante dos resultados apresentados as almôndegas de carne de capivara com diferentes concentrações de bacon poderiam ser ofertadas para o consumo da população. 


\section{REFERÊNCIAS}

ASSISTAT. Assistat - $\quad$ Assistência Estatística. 2000. Disponível em: <http://www.assistat.com/indexp.html>. Acesso em 12 fev. 2017.

BRASIL. INSTITUTO ADOLFO LUTZ (IAL). Métodos físico-químicos para análise de alimentos. ZENEBON, O.; PASCUET, N. S.; TIGLEA, P. (Coord.). 4.ed. 1.ed. Digital. São Paulo: Instituto Adolfo Lutz, 2008. 1020p.

BRASIL. Ministério da Agricultura, Pecuária e do Abastecimento. Secretaria de Inspeção de Produto Animal. Instrução Normativa no 62 de 26 de agosto de 2003. Oficializa os métodos analíticos oficiais para análises microbiológicas para controle de produtos de origem animal e água. Diário Oficial [da] República Federativa do Brasil. Brasília, DF, 18 de setembro de 2003. Seção 1, p. 14.

BRASIL. Ministério da Saúde. Agência Nacional de Vigilância Sanitária. Resolução nำ12, de 02 de janeiro de 2001. Aprova o Regulamento Técnico sobre Padrões Microbiológicos para Alimentos. Diário Oficial [da] Republica Federativa do Brasil. Brasília, DF, 10 de janeiro de 2001. Seção 1, p. 46-53.

FELIX, G. A. Comportamento alimentar e qualidade de carne de capivara (Hydrochoerus hydrochaeris Linnaeus, 1766) de vida livre, em áreas agrícolas. Dissertação (Mestrado em Zootecnia). Dourados, MS: Universidade Federal Da Grande Dourados, 2012. $81 \mathrm{f}$.

FOLETTO, T. C. Processo de abate e análises físico - químicas no frigorífico de suínos da Cotrijui (São Luiz Gonzaga/RS). Pelotas: Universidade Federal de Pelotas, 2007.

FRASSON, C.; SALGADO, J. M.; Animais silvestres: capivara - uma opção contra a fome e a deficiência em proteína animal. In: Simpósio Interface Nutrição X Agricultura. Piracicaba, 1990. Anais. Piracicaba: FEALQ, 1990. p. 175-200.

JARDIM, N. S. Sexo e diferentes pesos ao abate na qualidade da carne de capivara (Hydrochaeris hydrochaeris L.1766). 2001. 119p. Dissertação (Mestrado em Ciência dos alimentos) Universidade Federal de Lavras/UFLA, Lavras.

MIGUEL, G. Z. Caracterização da carne de capivara (Hyrdrochaereris hydrochaerys L. 1766) em idade adulta. (Dissertação), Lavras: Universidade Federal de Lavras, 2002.

PARDI, M.C.; SANTOS, I.F.; SOUZA, E.R.; PARDI, H.S. Ciência, higiene e tecnologia da carne: tecnologia da sua obtenção e transformação. Goiânia: Universidade Federal de Goias, v.1, 1993. $586 \mathrm{p}$.

REBELLO, F. P.; ODA, S. H. I.; BRESSAN, M. C.; VALLE, R. H. P.; MIGUEL, G.; BOARI, C.; MARQUES, S.; VIEIRA, J. O.; FARIA, P. B.; PISA, A. C. C. Qualidade microbiológica da carne de capivaras (Hydrochaeris hydrochaeris) submetidas a dois métodos de abate. XI Congresso de PósGraduação da UFLA/C. Alimentos. MG: Lavras, 2002.

ROCHA, D. C. C. Caracterização da cadeia produtiva de animais silvestres (capivaras, catetos e queixadas) no sul do Brasil. 2003. 243p. Dissertação (Mestrado em Agronegócios) - Universidade Federal do Rio Grande do Sul/ UFRGS, Porto Alegre. 
SARKIS, Flávia. Avaliação das condições microbiológicas de carnes de animais silvestres no município de São Paulo. 2002. 0 f. Dissertação (Mestrado) - Curso de Ciência e Tecnologia de Alimentos, Universidade de São Paulo, São Paulo, 2002.

SILVA, E. A Capivara: uma ampla revisão sobre este animal tão importante. Viçosa, MG: Universidade Federal de Viçosa, 2013. 